\title{
Polynomial Representation of Model Uncertainty in Dynamical Systems
}

\author{
Massimiliano Vasile
}

\begin{abstract}
This chapter introduces an approach to capture unmodelled components in dynamical systems through a hierarchical polynomial expansion in the state space. This approach is reminiscent of the empirical acceleration approach commonly used in precise orbit determination to account for unmodelled components in the force model.
\end{abstract}

\section{Introduction}

In orbit determination, and more generally in the propagation of uncertainty in dynamical systems, one problem is to capture uncertainties in the dynamical model itself. Although dynamical models are normally dependent on a number of parameters that can be calibrated using observations, the functional form of the dynamical model can be incomplete.

A commonly used approach, in precise orbit determination, is to introduce empirical accelerations as additional components of the dynamics. The value of these empirical accelerations can be defined in a number of different ways exploiting the available measurements.

It is customary to use time series expansions in polynomial or trigonometric form whose coefficients need to be found by matching the prediction of the model with the observations[1]. Another approach is to treat empirical accelerations as stochastic processes that can be reconstructed by a form of sequential filtering[2][3]. Other more recent approaches treat the unmodelled components as a stochastic process that is represented with a Gaussian mixture [4].

All these techniques generally work satisfactorily and allow one to use a reduced dynamics without the need for extremely high fidelity models. On the other hand

Massimiliano Vasile

University of Strathclyde, 75 Montrose Street, G11XJ, Glasgow, UK, e-mail: massimiliano.vasile@strath.ac.uk 
they do not immediately furnish a functional representation of the missing components. Even the use of time series expansions, which are valid within the interval in which the measurements are available, to extrapolate the behaviour of the dynamical system does not always lead to the desired results. Furthermore, time series do not provide information on the dependency of the empirical accelerations on any of the state variables.

For this reason, in this chapter, it is proposed the use of polynomial expansions, with unknown coefficients, of the unmodelled components in the state variables. If the state variables and the observations are treated as stochastic variables, then so are the coefficients of the polynomial expansion. It will be shown that this formulation can effectively capture missing components in simple dynamical systems, including hypersensitive ones, both in the case of a reduced number of observations and in the case of observations affected by uncertainty.

The chapter first introduces the general formulation of the problem and the polynomial expansion of the uncertain components. It then presents an optimisation process, that is required to calculate the coefficients of the expansion, and the concept of uncertainty distance as a metric in the space of the unknown coefficients. Some examples follow, that illustrate the results that can be obtained with this approach.

\section{Polynomial Expansion of Unmodelled Components}

Consider the two functions $f: S \times P \times\left[t_{0}: t_{0}+T\right] \longrightarrow \mathbb{R}^{n}$ and $v: S \times B \times\left[t_{0}: t_{0}+\right.$ $T] \longrightarrow \mathbb{R}^{n}$ with $S \subseteq \mathbb{R}^{n}$ and the initial value problem:

$$
\left\{\begin{array}{l}
\dot{s}=f(s, p, t)+v(s, b, t) \\
s\left(t_{0}\right)=s_{0}
\end{array}\right.
$$

where $s$ is the state vector. The function $v(s, b, t)$ represents some unknown function of the states that is capturing all unmodelled components, $p \in P \subseteq \mathbb{R}^{m_{p}}$ a set of uncertain model parameters, $b \in B \subseteq \mathbb{R}^{m_{b}}$ some unknown parameter vector of the unmodelled components, and $t$ the time coordinate. In this paper, we will study only the case in which the unmodelled components are not a function of time (the case with time dependence is easily obtained from the time independent formulation) and the missing component is added to the known component. Furthermore, let us consider the special case in which the function $v(s, b)$ can be expressed as follows:

$$
v(s, b)=\left\{\begin{array}{l}
0 \\
Q=\nabla_{r} U_{r}(s, b)+\nabla_{v} U_{v}(s, b)
\end{array}\right.
$$

where $Q: S \times B \times\left[t_{0}: t_{0}+T\right] \longrightarrow \mathbb{R}^{q}$, with $q<n$, and $U_{r}$ and $U_{v}$ are two continuous and differentiable scalar uncertainty functions that can be expanded in the following hierarchical form: 


$$
\begin{aligned}
& U_{r}(s, b) \simeq \sum_{i}^{2 N} a(b)_{i}^{r} \xi_{i}\left(s_{i}\right)+\sum_{i}^{2 N} \sum_{j}^{2 N} a(b)_{i j}^{r} \xi_{i j}\left(s_{i}, s_{j}\right)+ \\
& \sum_{i}^{2 N} \sum_{j}^{2 N} \sum_{k}^{2 N} a(b)_{i j k}^{r} \xi_{i j k}\left(s_{i}, s_{j}, s_{k}\right)+\ldots \\
& U_{v}(s, b) \simeq \sum_{i}^{2 N} a(b)_{i}^{v} \xi_{i}\left(s_{i}\right)+\sum_{i}^{2 N} \sum_{j}^{2 N} a(b)_{i j}^{v} \xi_{i j}\left(s_{i}, s_{j}\right)+ \\
& \sum_{i}^{2 N} \sum_{j}^{2 N} \sum_{k}^{2 N} a(b)_{i j k}^{v} \xi_{i j k}\left(s_{i}, s_{j}, s_{k}\right)+\ldots
\end{aligned}
$$

with $n=2 N$ the dimension of the state space. If Eqs.(1) describes the time evolution of a dynamical system, then $Q$ can be seen as a generalised force whose $h^{\text {th }}$ component, with $h=1, \ldots, q$, is:

$$
\begin{aligned}
& Q_{h}(s, b)=\frac{\partial U_{r}}{\partial r_{h}}+\frac{\partial U_{v}}{\partial v_{h}} \simeq c_{0}+\sum_{i}^{2 N} c(b)_{i} \zeta_{i}\left(s_{i}\right)+\sum_{i}^{2 N} \sum_{j}^{2 N} c(b)_{i j} \zeta_{i j}\left(s_{i}, s_{j}\right)+ \\
& \sum_{i}^{2 N} \sum_{j}^{2 N} \sum_{k}^{2 N} c(b)_{i j k} \zeta_{i j k}\left(s_{i}, s_{j}, s_{k}\right)+\ldots
\end{aligned}
$$

If $\zeta$ are monomial bases then the generalised forces reads:

$$
\begin{aligned}
& Q_{h}(s, b) \simeq c_{0}+\sum_{i \neq k}^{2 N} c(b)_{i} \Delta s_{i}+\sum_{i}^{2 N} \sum_{j}^{2 N} c(b)_{i j} \Delta s_{i} \Delta s_{j}+ \\
& \sum_{i}^{2 N} \sum_{j}^{2 N} \sum_{k}^{2 N} c(b)_{i j k} \Delta s_{i} \Delta s_{j} \Delta s_{k}+\ldots
\end{aligned}
$$

The vector $c$ has dimension $l$. Note that the vector function $Q$ can be directly expanded in polynomial series without going through a scalar function $U$. In fact, in the most general case in which the force field has no potential, it is easier to directly expand $Q$. However, in the case in which a $U$ function can be found, the uncertainty, from which the missing component is derived, can be expressed in a more compact form. Hence, although in the following we will use only $Q$, in this section we presented also the idea of deriving $Q$ from a scalar function $U$.

\subsection{Problem Statement}

Given $Q$ and a set of observations, one can obtain an approximated representation of the unmodelled components by finding the value of $c$ that best fits the measurements. Then, the value of the coefficients of expansion (4) can be obtained as the solution of an optimisation problem. The nature of the optimisation problem slightly differs depending on the integration scheme used to solve Eq. (1). If $N_{o}=l$ exact and distinct measurements are available then one needs to solve the following set of constraints:

$$
s\left(t_{i}, c\right)-s_{o}\left(t_{i}\right)=0 ; \quad 1=1, \ldots, N_{o}
$$

where $s\left(t_{i}, c\right)$ is the propagated state at time $t_{i}$ and $s_{o}\left(t_{i}\right)$ is the observed state at time $t_{i}$. If the number of observations $N_{o}$ is equal to the number of coefficients in the expansion (4), one could argue that the solution of problem (6) provides the exact values of all the coefficients $c$. If the number of exact measurements is lower than the number of coefficients $c$, a suitable smoothing function is required and the following problem needs to be solved: 


$$
\begin{gathered}
\min _{c} J(s, c) \\
\text { s.t. } \\
s\left(t_{i}, c\right)-s_{o}\left(t_{i}\right)=0 ; 1=1, \ldots, N_{o}
\end{gathered}
$$

where $J: S \times C \longrightarrow \mathbb{R}$ is a function of states $s$ and coefficients $c$. Note that, in general, problem (7) can have more than one solution for $c$ even when $N_{o}=l$. In fact, consider the simple second order differential linear equation:

$$
\ddot{x}=-k x+d
$$

where $k>0$ and $d>0$. The general solution has the form:

$$
x=A \cos (\omega t+\phi)+B
$$

Given that Eq. (8) has two unknowns, one could think that a single observation of both velocity and position would suffice. However, given the initial conditions $x(t=0)=0$ and $\dot{x}(t=0)=0$, for the observation $x=0$ and $\dot{x}=0$ at time $t=2 \pi$, a different solution exists for every $\omega=j \in \mathbb{N}^{+}$.

\subsection{Treatment of Stochastic Observations}

In the case of observations affected by an error, one cannot obtain a prediction of the exact value of the parameters $c$. In this case it is reasonable to assume that the initial conditions are also uncertain as they come from previous observations. If the expected values of the state vector, coming from observations, are enforced as hard constraints the result might not capture the actual missing components as the trajectory is forced to satisfy constraints that do not come from the natural dynamics but are dependent on the errors in the observations. One option is to consider the most probable value for each observation and a cost function that maximises the likelihood of correct identification. The other option is to quantify the uncertainty in the observations and initial conditions as confidence intervals on the observed states. More formally, consider the uncertainty space $(\Gamma, \mathscr{L}, \mathscr{M})$, with $\Gamma$ a non empty set, $\mathscr{L}$ a $\sigma$-algebra over $\Gamma$, and $\mathscr{M}$ an uncertain measure. Then the observed state $s_{o}$ is an uncertain variable $s_{o}:(\Gamma, \mathscr{L}, \mathscr{M}) \longrightarrow \mathbb{R}^{n}$. If the distribution of $s_{o}$ is available one can draw $N_{p}$ samples and solve problem (7) $N_{p}$ times to derive a distribution of the coefficients $c$. Alternatively, if no distribution is available for $s_{o}$ but $\Sigma$ is the collection of all the confidence intervals for all the observations, including the initial conditions, such that:

$$
\operatorname{Pr}\left(s_{o} \in \Sigma\right)>\varepsilon
$$

then one can formulate the following optimisation problem: 


$$
\begin{aligned}
& \min _{c \in \mathscr{C}} J(s, c) \\
& \text { s.t. } \\
& s\left(t_{i}\right) \in \Sigma \quad i=0, \ldots, N_{o}
\end{aligned}
$$

where $\varepsilon$ is an arbitrary value. The main advantage of this formulation is that no statistical moments are required and no exact distribution needs to be known a priori. Note that the initial conditions $s\left(t_{0}\right)$ are treated as an observed state.

\subsection{Uncertainty Distance}

The coefficients of the polynomial expansion define the motion of a physical system. Therefore, one assumption is that the dynamics will follow a minimum action principle. This is not necessarily always true as the missing components might correspond to a transient state. At the same time one can assume that the function $Q$ is a stochastic function that define the probability of the system to be in a particular state. As a consequence the function $J$ in (7) can be expressed in different forms. The one that is proposed in this paper assumes that the system is at a minimum energy state which means that the function $Q$ introduces the minimum level of noise compatible with the observations.

In this sense, the objective function in (7) can be interpreted as a distance in the metric vector space $\mathscr{C}$ of the parameters $c$. In this space, the origin represents the solution with no model uncertainty and any point at distance $\sqrt{c^{T} c}$ from the origin has uncertainty vector $Q$ and uncertainty distance:

$$
d_{u}=\int Q^{T} Q d t
$$

Note that by analogy, one can formulate problem (7) as the constrained optimal control problem:

$$
\begin{aligned}
& \min _{u} J(u)=\frac{1}{2} \int u^{2} d t \\
& \text { s.t. } \\
& \dot{s}=f(s, p, t)+u \\
& \text { and } \\
& s\left(t_{i}\right) \in \Sigma \quad i=0, \ldots, N_{o}
\end{aligned}
$$

where $u=Q$. It is important to note that other definitions of $J$ are possible, although the results in this paper seems to suggest that the $J$ function derived from (12) and defined as:

$$
J=c^{T} c
$$

provides good results, at least for the cases here analysed. 


\subsection{Solution through Optimisation}

Problem (11) can be solved using a simple multistart approach. Starting from a Latin Hypercube grid of randomly selected starting points we use the Matlab function fmincon to find a constrained local minimum from each of the points in the grid. The dynamics was integrated with the Matlab function ode 45 , with both the absolute and the relative tolerances set to 1e-9. In all the examples in this paper, this simple procedure was sufficient to find acceptable solutions. Given the global nature of the problem, more complex dynamical systems might require more sophisticated procedures. It should be noted that the convergence of fmincon was dependent on the scaling of the coefficients $c$. A wild choice for the first guess of $c$ or setting too broad boundaries for the $\mathscr{C}$ space can result in an integration failure of ode 45 .

\section{Examples}

This section contains a number of simple examples and the results that the proposed method can provide.

\subsection{Linear Elastic Dynamics with Friction}

The first example considers the following simple dynamical system with an elastic component and friction:

$$
\begin{aligned}
& \dot{v}=-x-0.5 v \\
& \dot{x}=v
\end{aligned}
$$

We assume that both elastic and friction components are unmodelled and we use the representation:

$$
\begin{aligned}
& \dot{v}=c_{1}+c_{2} x+c_{3} v+c_{4} v^{2} \\
& \dot{x}=v
\end{aligned}
$$

By introducing two measurements at $t=\pi$ and $t=\pi / 2$, assuming a uniform distribution of the measurements over an interval that is $\pm 10 \%$ of the exact value of the states, and solving problem (7) with cost function $J=c^{T} c$ we get the result in Figure 1. The figure shows in blue the mean and confidence interval of the value of the components of the $c$ vector. The same figure shows in red the exact value that the coefficient should have to reproduce the exact dynamics. As one can see the exact value is contained in the confidence interval and the estimated mean value is quite close to the exact one. In particular the first three coefficients are a good match. The fourth one has a much higher variance suggesting that more than one dynamics is compatible with the measurements. This is reasonable in this case as a small drag component, $c_{4} v^{2}$, would produce an effect similar to a larger friction component, $c_{3} v$, over the time span considered in this example. 


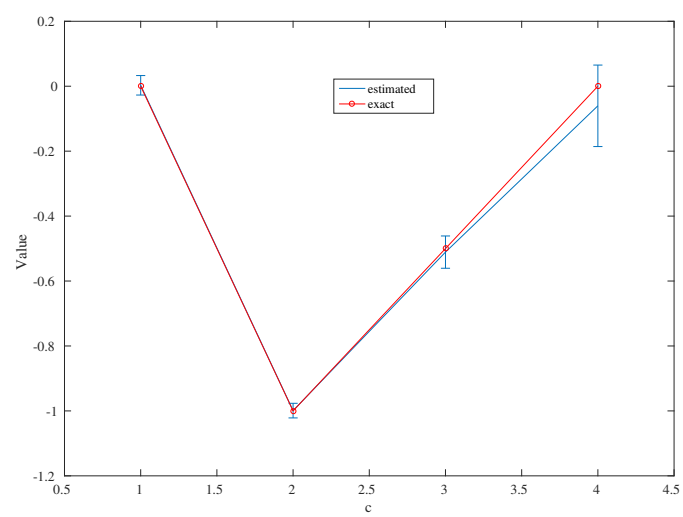

Fig. 1 Reconstructed coefficients for the linear elastic dynamics with friction. The dotted red curve is the exact value of the coefficients. The $x$-axis is the index of the coefficients and the y-axis their values.

\subsection{Orbital Motion with Unmodelled Drag}

The second example is an orbital motion with unknown drag component. The gravity component of the model is fully known but the observations show an additional component that is not modelled. The real dynamics is assumed to be governed by the following system of differential equations in polar coordinates:

$$
\begin{aligned}
& \dot{v_{r}}=-\frac{\mu}{r^{2}}+\frac{v_{t}^{2}}{r}-\frac{1}{2} \rho C_{d} v v_{r} \\
& \dot{v_{t}}=-\frac{v_{t} v_{r}}{r}-\frac{1}{2} \rho C_{d} v v_{t} \\
& \dot{r}=v_{r} \\
& \dot{\theta}=\frac{v_{t}}{r}
\end{aligned}
$$

We assume a unitary area to mass ratio, and a constant density $\rho$ such that the product of the density times the drag coefficient $C_{d}$ is $\rho C_{d}=10^{-6} \mathrm{~kg} / \mathrm{m}^{3}$. Furthermore, we assume that the expected trajectory, given the known dynamic components, is a circular orbit with $v_{r}(t=0)=v_{r_{0}}=0$ and $v_{t}(t=0)=v_{t_{0}}$. The orbital period, without drag, is $T=2 \pi \sqrt{r^{3} / \mu}$. If one expands the modulus of the velocity $v$ in Taylor series up to the first order, the differential equations with the drag term can be approximated as:

$$
\begin{aligned}
& \dot{v_{r}}=-\frac{\mu}{r^{2}}+\frac{v_{t}^{2}}{r}-\frac{1}{2} \rho C_{d} v_{t} v_{r} \\
& \dot{v_{t}}=-\frac{v_{t} v_{r}}{r}-\frac{1}{2} \rho C_{d} v_{t}^{2} \\
& \dot{r}=v_{r} \\
& \dot{\theta}=\frac{v_{t}}{r}
\end{aligned}
$$

In order to capture the unmodelled component of the dynamics, we assume the following expansion with terms up to order 2 in velocity and position: 


$$
\begin{aligned}
& \begin{array}{l}
v_{r}=-\frac{\mu}{r^{2}}+\frac{v_{t}^{2}}{r}+c_{1}+c_{3} r+c_{5} r^{2}+ \\
c_{7} r \theta+c_{9} v_{r}+c_{11} v_{r}^{2}+c_{13} v_{r} v_{t}
\end{array} \\
& \begin{array}{c}
\dot{v}_{t}=-\frac{v_{t} v_{r}}{r}+c_{2}+c_{4} \theta+c_{6} \theta^{2}+ \\
c_{8} r \theta+c_{10} v_{t}+c_{12} v_{t}^{2}+c_{14} v_{r} v_{t}
\end{array} \\
& \dot{r}=v_{r} \\
& \dot{\theta}=\frac{v_{t}}{r}
\end{aligned}
$$

If the linear effects in Eq. (18) are dominant over a given time span $\Delta t$, then the prediction given by Eq. (19) should be of the form:

$$
\begin{aligned}
& \dot{v_{r}}=-\frac{\mu}{r^{2}}+\frac{v_{t}^{2}}{r}+c_{13} v_{r} v_{t} \\
& \dot{v}_{t}=-\frac{v_{t} v_{r}}{r}+c_{12} v_{t}^{2} \\
& \dot{r}=v_{r} \\
& \dot{\theta}=\frac{v_{t}}{r}
\end{aligned}
$$

We can now introduce observations at time $t=T$ and $t=T / 2$, for a total of 8 constraint equations and 14 parameters, and solve problem (7) with cost function $J=c^{T} c$.



Fig. 2 Example of reconstructed gravity-drag dynamics for exact measurements

The estimated coefficients in the case of exact measurements are represented in Figure 2 (blue line) and compared to the expected values assuming a linear model (denoted by red circles). The resulting prediction of the trajectory over two orbits is shown in Figures 3 and 4. Note that the values of all the coefficients in the figures were scaled up by $10^{-6}$ to make them comparable to the value of general orbit perturbations. The $\mathscr{C}$ space in this case has boundaries $\left[-10^{-5}, 10^{-5}\right]$ for all the coefficients. As one can see, even if a linear model is assumed, the prediction of the 

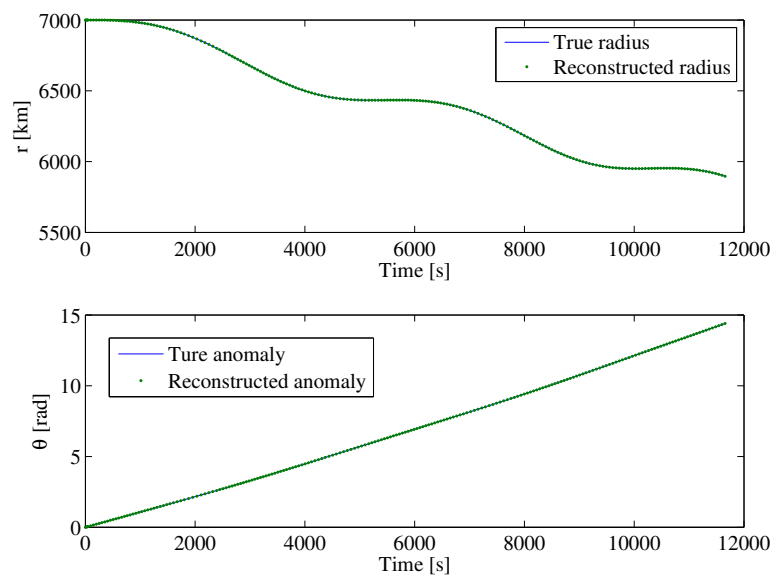

Fig. 3 Prediction over $2 T$ - radius and true anomaly
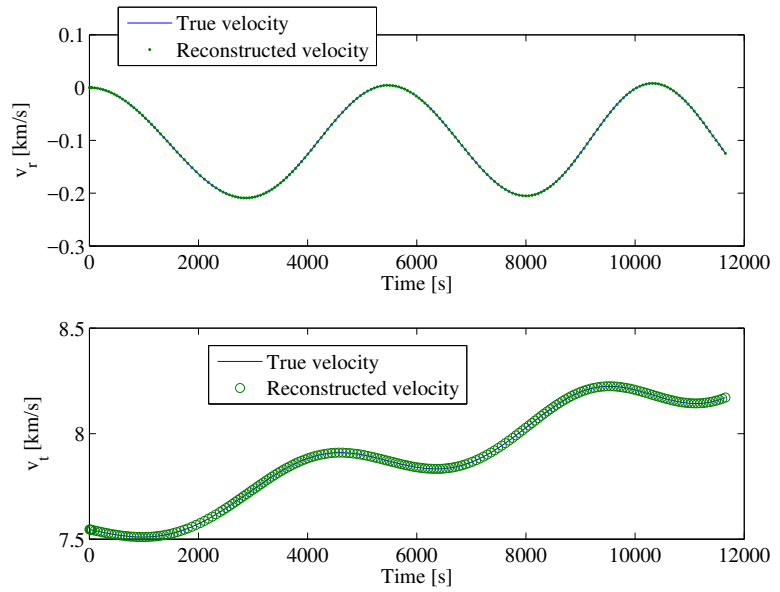

Fig. 4 Prediction over $2 T$ - velocity components

trajectory is very good over a horizon that is two times the interval over which the measurements are available.

If now one introduces the assumption that measurements are affected by an error, problem (11) needs to be solved under some assumptions on the initial conditions. The assumption in this paper is that the initial conditions are distributed uniformity over a given interval. The size of the confidence interval for the measurements is $10^{-4}$ of the measured value; accordingly the confidence interval on the initial conditions is set to the same value.

The estimated $c$ parameters are represented in Figure 5 together with their associated confidence intervals. As one can see, the expected value is close to the true solution. One thing that has to be taken into consideration is that the dynamics 


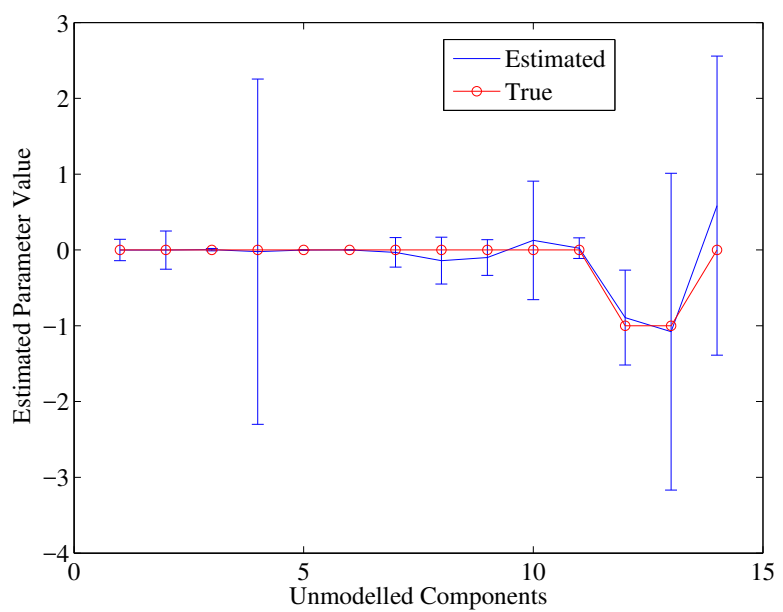

Fig. 5 Example of reconstructed gravity-drag dynamics with confidence intervals

that are simulated and measured are the true dynamics, not the linearised equations. Therefore, some components that are not in the linear model might be different from zero.

The other interesting result is that some components are nearly zero for every initial condition while other components, $c_{4}$ for example, have a wide variability. This result suggests that some components are irrelevant as they do not contradict the observations no matter which initial conditions are taken, while others substantially affect the evolution of the trajectory. Starting from this first iteration, one can then update the confidence intervals on the parameters $c$ and eventually converge to the correct missing components. Indeed since the uncertain function is based on a truncated series some components of the expansion might absorb the truncation error.

\subsection{Chaotic and Hypersensitive Systems}

As an example of chaotic dynamics and potentially hypersensitive optimal control problems, we analyse Duffing's equation:

$$
\begin{aligned}
& \dot{v}=-a x-d v-b x^{3}+g \cos (\omega t) \\
& \dot{x}=v
\end{aligned}
$$

with the following parameters: $a=1, d=0.1, b=0.1, g=1, \omega=1$. In this case the estimation is over $\Delta t=\pi / 4$ with the following expansion:

$$
\begin{aligned}
& \dot{v}=c_{1}+c_{2} x+c_{3} x^{2}+c_{4} v+c_{5} v^{2}+c_{6} x^{3}+g \cos (\omega t) \\
& \dot{x}=v
\end{aligned}
$$


In other words, the forcing term is known and is an input to the system, with the system itself a black box that needs to be identified. We first consider the case in which there are enough deterministic observations to compute all the $c$ parameters. In this case we directly solve problem (6) with 6 equispaced measurements. The result is represented in Figure 6. The dynamics is correctly reconstructed and the optimisation converges to a unique solution.

Considering the case in which there are only 4 deterministic measurements, we solve problem (7) with uncertainty distance metric $J=c^{T} c$. The result is shown in Figure 7 for a number of different and independent runs of the optimisation process. As one can see, multiple solutions exist that solve the constraints and minimise the cost function. The lowest cost function is reached when the estimated $c$ parameters approach the correct solution (shown as red circles).

Finally, we consider once again the case in which the measurements are equal to the number of coefficients but are stochastic. In this case the results are represented in Figure 8. Even in this case the confidence intervals contain the correct solution, but due to the hypersensitivity of the system an exact reconstruction of the missing components appears to be more difficult as a small variation in the initial conditions can correspond to a significantly different set of parameters. The $\varepsilon$ value for this case is $10^{-3}$ of the exact value of the states at the time of the measurement.



Fig. 6 Example of reconstructed Duffing's dynamics with exact observations and $N_{o}=2 N$ 


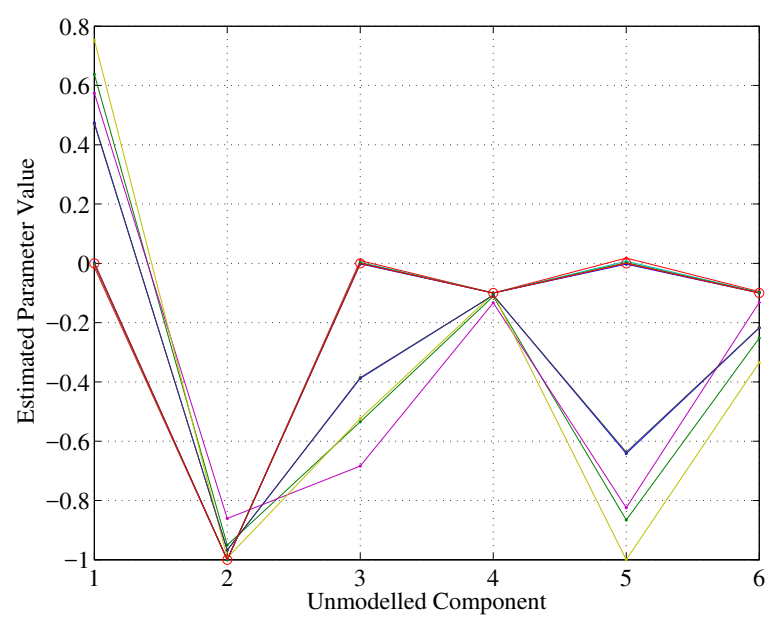

Fig. 7 Example of reconstructed Duffing's dynamics with exact observations and $N_{o}<2 N$

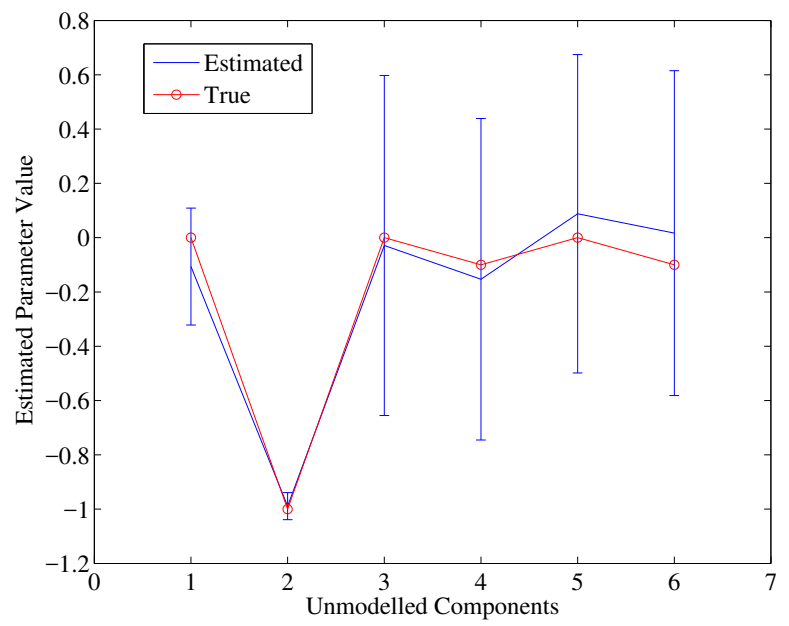

Fig. 8 Example of reconstructed Duffing's dynamics with confidence intervals

\section{Reachability Under Model Uncertainty}

Once the intervals for each of the parameters $c$ is available, one can solve a reachability problem under uncertainty. One can consider $Q$ to be a stochastic process comparable to a disturbance and the coefficients $c$ to be stochastic variables with probability space $(\Omega, \Gamma, \mathscr{P})$. In this section the problem is limited to reaching the target set given by a sphere centred in $r\left(s\left(t_{f}\right)\right)=0$ where $r(s)$ is the Euclidean distance. The reachability problem can be formulated as follows: 


$$
\begin{aligned}
& \min _{u} \max _{c} r\left(t_{f}\right) \\
& \text { s.t. } \\
& \dot{s}=f(s)+Q(s, c)+u \\
& s_{0} \in \Sigma_{0} \\
& u \in \mathscr{U} \\
& c \in \Omega \subseteq \mathscr{C}
\end{aligned}
$$

where $\mathscr{U}$ is the control space, $\Omega$ is a subspace of $\mathscr{C}$ defined by the confidence intervals for all the parameters $c$ and $\Sigma_{0}$ is the set of initial conditions. As an example we can consider problem (15) where the confidence intervals for the parameters $c$ are available from the previous identification process and one wants the optimal control $u$ that satisfies:

$$
\begin{aligned}
& \min _{u} \max _{c} x^{2}\left(t_{f}\right) \\
& \text { s.t. } \\
& \ddot{x}=c_{0}+c_{1} x+c_{2} x^{2}+c_{3} v^{2}+u \\
& x_{0} \in \Sigma_{0} \\
& u \in[-0.1,0.1] \\
& c \in \Omega \subseteq \mathscr{C}
\end{aligned}
$$

The control is represented with cubic splines collocated at ten regular points in time in the interval $\left[0, t_{f}\right]$. Additionally, the control is bounded to be in the interval $[-0.1,0.1]$. Problem (24) can be solved with the algorithm presented in [5] for the solution of minmax problems. The optimisation converges in a couple of iterations with the result in Figures 9. Figures 9 show the min-max solution compared to the solution with no control and worst case disturbance and the solution with no disturbance and the control action derived from the solution of problem (24).

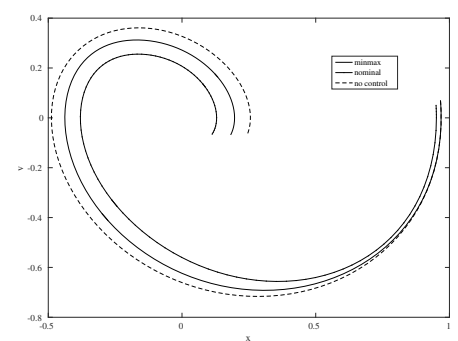

(a)

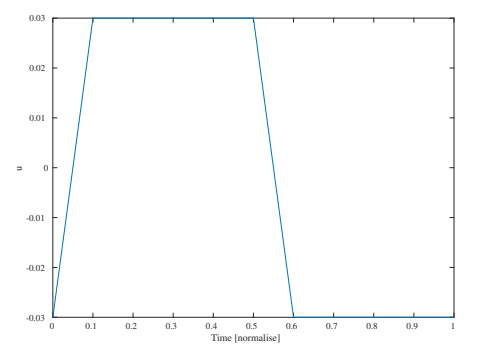

(b)

Fig. 9 a) Phase space representation of the min-max solution; b) Control profile for the min-max solution 


\section{Final Remarks}

The chapter presented an approach to reconstruct unmodelled components in dynamical systems by using hierarchical polynomial expansions. It was shown that the expansion proposed in this paper can be derived from a scalar uncertainty function with the form of a pseudo-potential. Other choices are possible to include effects that can not be reduced to a scalar potential function.

In all the cases analysed in this chapter, the approach provided a good estimation of the missing components in the dynamical model. The next step is to iterate over longer arcs and to update the estimation when new measurements are available. The chapter proposed also the solution of a reachability problem where the unmodelled component are a stochastic disturbance that needs to be controlled by an optimal control action. The problem translates into a min-max optimisation that provides an optimal, yet robust, solution.

Acknowledgements This work is partially supported by the JSPS fellowship number L15548 and by the FP7 MCA Stardust ITN.

\section{References}

1. M. Alessi, E., S. Cicalo, and A. Milani. Accelerometer data hanfling for the bepicolombo orbit determination. In Advances in the Astronautical Society 145 AAS 12-309 1st IAA Conference on Dynamics and Control of Space Systems, 2012.

2. O. Montenbruck, T. Helleputte, R. Kroes, and E Gill. Reduced dynamic orbit determination using gps code and carrier measurements. Aerospace Science and Technology, 9:261-271, 2005.

3. M. Vetrisano, C. Colombo, and M. Vasile. Asteroid rotation and orbit control via laser ablation. Advances in Space Research, 9, 2015.

4. D. Giza, P. Singla, and M. Jah. An approach for nonlinear uncertainty propagation: Application to orbital mechanics. In Paper AIAA 2009-6082, AIAA Guidance, Navigation, and Control Conference 10 - 13 August 2009, Chicago, Illinois.

5. M. Vasile. On the solution of min-max problems in robust optimization. In Proceedings of EVOLVE2014, Bejing, 2014. 